\title{
Common Fixed Points in a Partially Ordered Partial Metric Space
}

\author{
Daniela Paesano and Pasquale Vetro \\ Dipartimento di Matematica e Informatica, Università Degli Studi di Palermo, Via Archirafi, 34-90123 Palermo, Italy \\ Correspondence should be addressed to Pasquale Vetro; vetro@math.unipa.it
}

Received 29 August 2012; Revised 12 September 2012; Accepted 17 September 2012

Academic Editor: Harumi Hattori

Copyright (C) 2013 D. Paesano and P. Vetro. This is an open access article distributed under the Creative Commons Attribution License, which permits unrestricted use, distribution, and reproduction in any medium, provided the original work is properly cited.

In the first part of this paper, we prove some generalized versions of the result of Matthews in (Matthews, 1994) using different types of conditions in partially ordered partial metric spaces for dominated self-mappings or in partial metric spaces for self-mappings. In the second part, using our results, we deduce a characterization of partial metric 0 -completeness in terms of fixed point theory. This result extends the Subrahmanyam characterization of metric completeness.

\section{Introduction}

In the mathematical field of domain theory, attempts were made in order to equip semantics domain with a notion of distance. In particular, Matthews [1] introduced the notion of a partial metric space as a part of the study of denotational semantics of data for networks, showing that the contraction mapping principle can be generalized to the partial metric context for applications in program verification. Moreover, the existence of several connections between partial metrics and topological aspects of domain theory has been lately pointed by other authors as O'Neill [2], Bukatin and Scott [3], Bukatin and Shorina [4], Romaguera and Schellekens [5], and others (see also [6-14] and the references therein).

After the result of Matthews [1], the interest for fixed point theory developments in partial metric spaces has been constantly growing, and many authors presented significant contributions in the directions of establishing partial metric versions of well-known fixed point theorems for the existence of fixed points, common fixed points, and coupled fixed points in classical metric spaces (see e.g., $[15,16])$. Obviously, we cannot cite all these papers but we give only a partial list [17-49].

Recently, Romaguera [50] proved that a partial metric space $(X, d)$ is 0 -complete if and only if every $p^{s}$-Caristi mapping on $X$ has a fixed point. In particular, the result of Romaguera extended Kirk's [51] characterization of metric completeness to a kind of complete partial metric spaces.
Successively, Karapinar in [36] extended the result of Caristi and Kirk [52] to partial metric spaces.

In the first part of this paper, following this research direction, we prove some generalized versions of the result of Matthews by using different types of conditions in ordered partial metric spaces for dominated self-mappings or in partial metric spaces for self-mappings. The notion of dominated mapping of economics, finance, trade, and industry is also applied to approximate the unique solution of nonlinear functional equations. In the second part, using the results obtained in the first part, we deduce a characterization of partial metric 0-completeness in terms of fixed point theory. This result extends the Subrahmanyam [53] characterization of metric completeness. For other characterizations of metric completeness in terms of fixed point theory, the reader can see, for example, $[54,55]$ and for partial metric completeness, [41].

\section{Preliminaries}

First, we recall some definitions and some properties of partial metric spaces that can be found in $[1,2,40,48,50]$. A partial metric on a nonempty set $X$ is a function $p: X \times X \rightarrow$ $[0,+\infty)$ such that for all $x, y, z \in X$

$$
\begin{aligned}
& \left(p_{1}\right) x=y \Leftrightarrow p(x, x)=p(x, y)=p(y, y), \\
& \left(p_{2}\right) p(x, x) \leq p(x, y),
\end{aligned}
$$




$$
\begin{aligned}
& \left(p_{3}\right) p(x, y)=p(y, x), \\
& \left(p_{4}\right) p(x, y) \leq p(x, z)+p(z, y)-p(z, z) .
\end{aligned}
$$

A partial metric space is a pair $(X, p)$ such that $X$ is a nonempty set and $p$ is a partial metric on $X$. It is clear that if $p(x, y)=0$, then from $\left(p_{1}\right)$ and $\left(p_{2}\right)$, it follows that $x=y$. But if $x=y, p(x, y)$ may not be 0 . A basic example of a partial metric space is the pair $([0,+\infty), p)$, where $p(x, y)=$ $\max \{x, y\}$ for all $x, y \in[0,+\infty)$. Other examples of partial metric spaces which are interesting from a computational point of view can be found in [1].

Each partial metric $p$ on $X$ generates a $T_{0}$ topology $\tau_{p}$ on $X$ which has as a base the family of open $p$-balls $\left\{B_{p}(x, \varepsilon)\right.$ : $x \in X, \varepsilon>0\}$, where

$$
B_{p}(x, \varepsilon)=\{y \in X: p(x, y)<p(x, x)+\varepsilon\}
$$

for all $x \in X$ and $\varepsilon>0$.

If $p$ is a partial metric on $X$, then the function $p^{s}: X \times$ $X \rightarrow[0,+\infty)$ given by

$$
p^{s}(x, y)=2 p(x, y)-p(x, x)-p(y, y)
$$

is a metric on $X$.

Let $(X, p)$ be a partial metric space. A sequence $\left\{x_{n}\right\}$ in $(X, p)$ converges to a point $x \in X$ if and only if $p(x, x)=$ $\lim _{n \rightarrow+\infty} p\left(x, x_{n}\right)$.

A sequence $\left\{x_{n}\right\}$ in $(X, p)$ is called a Cauchy sequence if there exists (and is finite) $\lim _{n, m \rightarrow+\infty} p\left(x_{n}, x_{m}\right)$.

A partial metric space $(X, p)$ is said to be complete if every Cauchy sequence $\left\{x_{n}\right\}$ in $X$ converges, with respect to $\tau_{p}$, to a point $x \in X$ such that $p(x, x)=\lim _{n, m \rightarrow+\infty} p\left(x_{n}, x_{m}\right)$.

A sequence $\left\{x_{n}\right\}$ in $(X, p)$ is called 0 -Cauchy if $\lim _{n, m \rightarrow+\infty} p\left(x_{n}, x_{m}\right)=0$. We say that $(X, p)$ is 0 -complete if every 0 -Cauchy sequence in $X$ converges, with respect to $\tau_{p}$, to a point $x \in X$ such that $p(x, x)=0$.

On the other hand, the partial metric space $(\mathbb{Q} \cap$ $[0,+\infty), p)$, where $\mathbb{Q}$ denotes the set of rational numbers and the partial metric $p$ is given by $p(x, y)=\max \{x, y\}$, provides an example of a 0 -complete partial metric space which is not complete.

It is easy to see that every closed subset of a complete partial metric space is complete.

Lemma 1 (see $[1,40])$. Let $(X, p)$ be a partial metric space. Then

(a) $\left\{x_{n}\right\}$ is a Cauchy sequence in $(X, p)$ if and only if it is a Cauchy sequence in the metric space $\left(X, p^{s}\right)$.

(b) A partial metric space $(X, p)$ is complete if and only if the metric space $\left(X, p^{s}\right)$ is complete. Furthermore, $\lim _{n \rightarrow+\infty} p^{s}\left(x_{n}, x\right)=0$ if and only if

$$
p(x, x)=\lim _{n \rightarrow+\infty} p\left(x_{n}, x\right)=\lim _{n, m \rightarrow+\infty} p\left(x_{n}, x_{m}\right) \text {. }
$$

The following lemma is obvious.

Lemma 2. Let $(X, p)$ be a partial metric space and $\left\{x_{n}\right\} \subset X$. If $x_{n} \rightarrow x \in X$ and $p(x, x)=0$, then $\lim _{n \rightarrow+\infty} p\left(x_{n}, z\right)=$ $p(x, z)$ for all $z \in X$.
Define $p(x, A)=\inf \{p(x, a): a \in A\}$. Then $a \in \bar{A} \Leftrightarrow$ $p(a, A)=p(a, a)$, where $\bar{A}$ denotes the closure of $A$ (for details see [22, Lemma 1]). From

$$
p^{s}(x, a)=2 p(x, a)-p(x, x)-p(a, a) \leq 2 p(x, a)
$$

for every $a \in A$, we deduce that $p^{s}(x, A) \leq 2 p(x, A)$.

Let $X$ be a nonempty set and $T, f: X \rightarrow X$. The mappings $T, f$ are said to be weakly compatible if they commute at their coincidence points (i.e., $T f x=f T x$ whenever $T x=f x$ ). A point $y \in X$ is called a point of coincidence of $T$ and $f$ if there exists a point $x \in X$ such that $y=T x=f x$.

Lemma 3 (see [56]). Let $X$ be a nonempty set and the mappings $T, f: X \rightarrow X$ have a unique point of coincidence $v$ in $X$. If $T$ and $f$ are weakly compatible, then $T$ and $f$ have a unique common fixed point.

Let $X$ be a nonempty set. If $(X, p)$ is a partial metric space and $(X, \preceq)$ is a partially ordered set, then $(X, p, \preceq)$ is called a partially ordered partial metric space. $x, y \in X$ are called comparable if $x \preceq y$ or $y \preceq x$ holds. Let $(X, \preceq)$ be a partially ordered set and $T, f: X \rightarrow X$ two mappings. $T$ is called an $f$-dominated mapping if $T x \preceq f x$ for every $x \in X$.

\section{Main Results}

Let $(X, p)$ be a partial metric space and $T, f: X \rightarrow X$ be such that $T X \subset f X$. For every $x_{0} \in X$ we consider the sequence $\left\{x_{n}\right\} \subset X$ defined by $f x_{n}=T x_{n-1}$ for all $n \in \mathbb{N}$ and we say that $\left\{T x_{n}\right\}$ is a $T$ - $f$-sequence of the initial point $x_{0}$ (see [57]).

Denote with $\Psi$ the family of non-decreasing functions $\psi$ : $[0,+\infty) \rightarrow[0,+\infty)$ such that $\psi(t)>0$ and $\lim _{n \rightarrow+\infty} \psi^{n}(t)=$ 0 for each $t>0$, where $\psi^{n}$ is the $n$th iterate of $\psi$.

Lemma 4. For every function $\psi \in \Psi$, the following holds, iffor each $t>0, \lim _{n \rightarrow+\infty} \psi^{n}(t)=0$ then $\psi(t)<t$.

The following theorem is one of our main results, and it ensures the existence of a common fixed point for two selfmappings in the setting of partially ordered partial metric spaces.

Theorem 5. Let $(X, p, \preceq)$ be a partially ordered partial metric space and $T, f: X \rightarrow X$ two mappings such that $T X \subset f X$. Assume that there exists $\psi \in \Psi$ such that

$$
\begin{aligned}
& p(T x, T y) \\
& \quad \leq \max \{\psi(p(f x, f y)), \psi(p(f x, T x)), \psi(p(f y, T y))\}
\end{aligned}
$$

for all $x, y \in X$ with $f x$ and $f y$ comparable. If the following conditions hold:

(i) $T$ is a $f$-dominated mapping,

(ii) either $T X$ or $f X$ is a 0 -complete subspace of $X$, 
(iii) for a non-increasing sequence $\left\{f x_{n}\right\} \subset X$ converging to $f u \in X$, we have $f u \preceq f x_{n}$ for all $n \in \mathbb{N}$ and $f f u \preceq f u$,

then $T$ and $f$ have a point of coincidence. Moreover, if $T$ and $f$ are weakly compatible, then $T$ and $f$ have a common fixed point.

Proof. Let $x_{0} \in X$ be fixed and $\left\{T x_{n}\right\}$ be a $T$ - $f$-sequence of the initial point $x_{0}$. As $f x_{n+1}=T x_{n} \leq f x_{n}$ for all $n \in \mathbb{N}$, then the sequence $\left\{T x_{n}\right\}$ is non-increasing.

If $T x_{n}=T x_{n-1}=f x_{n}$ for some $n \in \mathbb{N}$, then $y=$ $T x_{n}=f x_{n}$ is a point of coincidence of $T$ and $f$. Suppose that $T x_{n} \neq T x_{n-1}$ for all $n \in \mathbb{N}$. Since $f x_{n}$ and $f x_{n+1}$ are comparable for all $n \in \mathbb{N}$, we have

$$
\begin{gathered}
p\left(T x_{n+1}, T x_{n}\right) \\
\leq \max \left\{\psi\left(p\left(f x_{n+1}, f x_{n}\right)\right), \psi\left(p\left(f x_{n+1}, T x_{n+1}\right)\right)\right. \\
\left.\psi\left(p\left(f x_{n}, T x_{n}\right)\right)\right\} \\
=\max \left\{\psi\left(p\left(T x_{n}, T x_{n-1}\right)\right), \psi\left(p\left(T x_{n+1}, T x_{n}\right)\right)\right\} \\
\text { If } \quad \max \left\{\psi\left(p\left(T x_{n}, T x_{n-1}\right)\right), \psi\left(p\left(T x_{n+1}, T x_{n}\right)\right)\right\} \\
\psi\left(p\left(T x_{n+1}, T x_{n}\right)\right) \text { from } \\
p\left(T x_{n+1}, T x_{n}\right) \leq \psi\left(p\left(T x_{n+1}, T x_{n}\right)\right) \\
<p\left(T x_{n+1}, T x_{n}\right)
\end{gathered}
$$

we obtain a contradiction and so

$$
\begin{aligned}
\max & \left\{\psi\left(p\left(T x_{n}, T x_{n-1}\right)\right), \psi\left(p\left(T x_{n+1}, T x_{n}\right)\right)\right\} \\
& =\psi\left(p\left(T x_{n}, T x_{n-1}\right)\right) .
\end{aligned}
$$

Then, we have

$$
p\left(T x_{n+1}, T x_{n}\right) \leq \psi^{n}\left(p\left(T x_{1}, T x_{0}\right)\right), \quad \forall n \in \mathbb{N}
$$

and hence

$$
\lim _{n \rightarrow+\infty} p\left(T x_{n+1}, T x_{n}\right)=0 .
$$

Fix $\varepsilon>0$ and we choose $n(\varepsilon) \in \mathbb{N}$ such that

$$
p\left(T x_{m}, T x_{m+1}\right)<\varepsilon-\psi(\varepsilon)
$$

for all $m \geq n(\varepsilon)$. Let $m \geq n(\varepsilon)$ and we show that

$$
p\left(T x_{m}, T x_{n+1}\right)<\varepsilon, \quad \forall n \geq m .
$$

Clearly, (12) is true for $n=m$. Suppose that (12) holds for some $n \geq m$, as $f x_{i}$ and $f x_{j}$ are comparable for all $i, j \in \mathbb{N}$, then

$$
\begin{aligned}
& p\left(T x_{m}, T x_{n+2}\right) \\
& \leq p\left(T x_{m}, T x_{m+1}\right)+p\left(T x_{m+1}, T x_{n+2}\right) \\
& \leq p\left(T x_{m}, T x_{m+1}\right) \\
& \quad+\max \left\{\psi\left(p\left(T x_{m}, T x_{n+1}\right)\right), \psi\left(p\left(T x_{m}, T x_{m+1}\right)\right),\right. \\
& \left.\psi\left(p\left(T x_{n+1}, T x_{n+2}\right)\right)\right\} \\
& <\varepsilon-\psi(\varepsilon)+\max \{\psi(\varepsilon), \psi(\varepsilon-\psi(\varepsilon))\}=\varepsilon .
\end{aligned}
$$

This implies that (12) holds for $n+1$ and by induction, it holds for all $n \geq m$. From (12), we deduce that there exists

$$
\lim _{n, m \rightarrow+\infty} p\left(T x_{n}, T x_{m}\right)=0
$$

and hence $\left\{T x_{n}\right\}$ is a 0 -Cauchy sequence.

Suppose that $T X$ is a 0 -complete subspace of $(X, p)$, then there exists $y \in T X \subset f X$ such that

$$
p(y, y)=\lim _{n \rightarrow+\infty} p\left(T x_{n}, y\right)=\lim _{n, m \rightarrow+\infty} p\left(T x_{n}, T x_{m}\right)=0 .
$$

This holds also if $f X$ is 0 -complete with $y \in f X$.

Let $u \in X$ be such that $y=f u$. We show that $y$ is a point of coincidence of $T$ and $f$. If not, we have $p(f u, T u)>0$. This implies that there exists $n_{0} \in \mathbb{N}$ such that

$$
\begin{aligned}
\max \{\psi( & \left.\left.\left(T x_{n-1}, f u\right)\right), \psi\left(p\left(T x_{n-1}, T x_{n}\right)\right), \psi(p(f u, T u))\right\} \\
& =\psi(p(f u, T u)),
\end{aligned}
$$

for every $n \geq n_{0}$. By condition (iii), $f x_{n}$ and $f u$ are comparable for every $n \in \mathbb{N}$ and hence, by condition (5) with $x=x_{n}$ and $y=u$, we deduce that

$$
\begin{aligned}
& p\left(T x_{n}, T u\right) \\
& \leq \max \left\{\psi\left(p\left(f x_{n}, f u\right)\right), \psi\left(p\left(f x_{n}, T x_{n}\right)\right),\right. \\
& \psi(p(f u, T u))\} \\
& =\max \left\{\psi\left(p\left(T x_{n-1}, f u\right)\right), \psi\left(p\left(T x_{n-1}, T x_{n}\right)\right),\right. \\
& \psi(p(f u, T u))\} \\
& =\psi(p(f u, T u)),
\end{aligned}
$$

for every $n \geq n_{0}$. Letting $n \rightarrow+\infty$ in the previous inequality and using Lemma 2, we obtain

$$
p(f u, T u) \leq \psi(p(f u, T u))<p(f u, T u),
$$

which implies $p(f u, T u)=0$, that is, $T u=f u$. Thus, we have shown that $y=f u=T u$ is a point of coincidence of $T$ and $f$. If $T$ and $f$ are weakly compatible, then $T y=T f u=f T u=$ $f y$. By condition (iii), $f y=f f u \preceq f u$, that is, $f y$ and $f u$ are comparable. Using the contractive condition (5), we get

$$
\begin{aligned}
& p(T u, T y) \\
& \quad \leq \max \{\psi(p(f u, f y)), \psi(p(f u, T u)), \psi(p(f y, T y))\} \\
& \quad=\psi(p(f u, f y))<p(f u, f y),
\end{aligned}
$$

which implies $T y=T u=y$ and hence $y$ is a common fixed point of $T$ and $f$.

We shall give a sufficient condition for the uniqueness of the common fixed point in Theorem 5 .

Theorem 6. Let all the conditions of Theorem 5 be satisfied. If the following condition holds: 
(iv) for all $x, y \in f X$ there exists $v_{0} \in X$ such that $f v_{0} \preceq$ $x, f v_{0} \preceq y$ and $\lim _{n \rightarrow+\infty} p\left(T v_{n-1}, T v_{n}\right)=0$, where $\left\{T v_{n}\right\}$ is the $T$ - $f$-sequence of the initial point $v_{0}$,

then $T$ and $f$ have a unique common fixed point.

Proof. Let $z, w$ be two common fixed points of $T$ and $f$ with $z \neq w$. If $z$ and $w$ are comparable, then using the contractive condition (5), we deduce that $z=w$. If $z$ and $w$ are not comparable, then there exists $v_{0} \in X$ such that $f v_{0} \preceq z=f z$, $f v_{0} \preceq w=f w$. As $T$ is a $f$-dominated mapping, we get that

$$
f v_{1}=T v_{0} \preceq f v_{0} \preceq z=f z .
$$

To continue, we obtain

$$
f v_{n+1}=T v_{n} \preceq f v_{n} \preceq z=f z \quad \forall n \in \mathbb{N}
$$

and hence $f v_{n}$ and $f z$ are comparable.

Using the contractive condition (5) with $x=v_{n}$ and $y=$ $z$, we get

$$
\begin{aligned}
& p\left(T v_{n}, T z\right) \\
& \leq \max \left\{\psi\left(p\left(f v_{n}, f z\right)\right), \psi\left(p\left(f v_{n}, T v_{n}\right)\right),\right. \\
&\psi(p(f z, T z))\} \\
&=\max \left\{\psi\left(p\left(T v_{n-1}, T z\right)\right), \psi\left(p\left(T v_{n-1}, T v_{n}\right)\right),\right. \\
&\psi(p(T z, T z))\}
\end{aligned}
$$

for all $n \in \mathbb{N}$. Since, the contractive condition (5) ensures that $p(T z, T z)=0$, we have

$$
p\left(T v_{n}, T z\right) \leq \max \left\{\psi\left(p\left(T v_{n-1}, T z\right)\right), \psi\left(p\left(T v_{n-1}, T v_{n}\right)\right)\right\} .
$$

Now, by condition (iv), $\lim _{n \rightarrow+\infty} p\left(T v_{n-1}, T v_{n}\right)=0$ and hence for $n$ sufficiently large, we have

$$
p\left(T v_{n}, T z\right) \leq \psi\left(p\left(T v_{n-1}, T z\right)\right) .
$$

Without loss of generality, assuming that (24) holds for all $n \in$ $\mathbb{N}$, it follows that

$$
p\left(T v_{n}, T z\right) \leq \psi^{n}\left(p\left(T v_{0}, T z\right)\right) .
$$

Now, letting $n \rightarrow+\infty$ in (25), we obtain

$$
\lim _{n \rightarrow+\infty} p\left(T v_{n}, T z\right)=0 \text {. }
$$

With similar arguments, we deduce that $\lim _{n \rightarrow+\infty} p\left(T w, T v_{n}\right)=0$. Hence

$$
\begin{aligned}
0 & <p(w, z)=p(T w, T z) \\
& \leq p\left(T w, T v_{n}\right)+p\left(T v_{n}, T z\right) \longrightarrow 0
\end{aligned}
$$

as $n \rightarrow+\infty$, which is a contradiction. Thus $T$ and $f$ have a unique common fixed point. result.
Theorem 7. Let $(X, p)$ be a partial metric space and $T, f$ : $X \rightarrow X$ two mappings such that $T X \subset f X$. Assume that there exists $\psi \in \Psi$ such that

$$
\begin{aligned}
& p(T x, T y) \\
& \quad \leq \max \{\psi(p(f x, f y)), \psi(p(f x, T x)), \psi(p(f y, T y))\}
\end{aligned}
$$

for all $x, y \in X$. If TX or $f X$ is a 0 -complete subspace of $X$, then $T$ and $f$ have a unique point of coincidence. Moreover, if $T$ and $f$ are weakly compatible, then $T$ and $f$ have a unique common fixed point.

Proof. Proceeding as in the proof of Theorem 5, we get that $T$ and $f$ have a unique point of coincidence and, by Lemma 3, $T$ and $f$ have a unique common fixed point.

From Theorem 7, we can deduce the following corollaries.

Corollary 8 (Banach type). Let $(X, p)$ be a partial metric space and $T, f: X \rightarrow X$ two mappings such that $T X \subset f X$. Assume that

$$
p(T x, T y) \leq k p(f x, f y)
$$

for all $x, y \in X$, where $0 \leq k<1$. If TX or $f X$ is a 0 -complete subspace of $X$, then $T$ and $f$ have a unique point of coincidence. Moreover, if $T$ and $f$ are weakly compatible, then $T$ and $f$ have a unique common fixed point.

Corollary 9 (Bianchini type). Let $(X, p)$ be a partial metric space and let $T, f: X \rightarrow X$ be two mappings such that $T X \subset$ $f X$. Assume that

$$
p(T x, T y) \leq k \max \{p(f x, T x), p(f y, T y)\}
$$

for all $x, y \in X$, where $0 \leq k<1$. If TX or $f X$ is a 0 -complete subspace of $X$, then $T$ and $f$ have a unique point of coincidence. Moreover, if $T$ and $f$ are weakly compatible, then $T$ and $f$ have a unique common fixed point.

Corollary 10 (Reich type [58]). Let $(X, p)$ be a partial metric space and let $T, f: X \rightarrow X$ be two mappings such that $T X \subset$ $f X$. Assume that

$$
p(T x, T y) \leq a p(f x, f y)+b p(f x, T x)+c p(f y, T y)
$$

for all $x, y \in X$, where $a, b, c \geq 0$ and $a+b+c<1$. If $T X$ or $f X$ is a 0 -complete subspace of $X$, then $T$ and $f$ have a unique point of coincidence. Moreover, if $T$ and $f$ are weakly compatible, then $T$ and $f$ have a unique common fixed point.

The following example shows that there exist mappings that satisfy the contractive condition (28), but are not quasicontractions [59].

Example 11. Consider the set $X=\{1,2,3\}$ and the function $p: X \times X \rightarrow[0,+\infty)$ given by $p(1,2)=p(2,3)=$ $1, p(1,3)=3 / 2, p(1,1)=p(3,3)=1 / 2, p(2,2)=0$, 
and $p(x, y)=p(y, x)$. Obviously, $p$ is a partial metric on $X$, but it is not a metric (since $p(x, x) \neq 0$ for $x=1$ and $x=3)$. Clearly, $(X, p)$ is a 0 -complete partial metric space. Let $T, f: X \rightarrow X$ be defined by $T 1=2, T 2=2, T 3=1$ and $f x=x$ for every $x \in X$. Take $\psi(t)=(2 / 3) t$ for every $t \geq 0$.

First, we will check that $T$ and $f$ satisfy the contractive condition (28). If $x, y \in\{1,2\}$, then $p(T x, T y)=p(2,2)=0$ and (28) trivially holds. Let, for example, $y=3$, then we have the following three cases:

$$
\begin{aligned}
p(T 1, T 3) & =p(2,1)=1 \leq \frac{2}{3} \cdot \frac{3}{2} \\
& =\max \{\psi(p(1,3)), \psi(p(1,2)), \psi(p(3,1))\} \\
p(T 2, T 3) & =p(2,1)=1 \leq \frac{2}{3} \cdot \frac{3}{2} \\
& =\max \{\psi(p(2,3)), \psi(p(2,2)), \psi(p(3,1))\} \\
p(T 3, T 3) & =p(1,1)=\frac{1}{2}<\frac{2}{3} \cdot \frac{3}{2} \\
& =\max \{\psi(p(3,3)), \psi(p(3,1))\} .
\end{aligned}
$$

Thus, all the conditions of Theorem 7 are satisfied and the existence of a common fixed point of $T$ and $f$ (which is 2) follows. The same conclusion cannot be obtained by the main results from [59]. Indeed, using $p^{s}(a, b)=2 p(a, b)-p(a, a)-$ $p(b, b)$, and then taking $p^{s}$ instead of $p, x=1, y=3$ in (5), we obtain

$$
\begin{gathered}
L=p^{s}(T 1, T 3)=p^{s}(2,1)=\frac{3}{2}, \\
R=\max \left\{\psi\left(p^{s}(1,3)\right), \psi\left(p^{s}(1, T 1)\right), \psi\left(p^{s}(3, T 3)\right),\right. \\
\left.\psi\left(p^{s}(1, T 3)\right), \psi\left(p^{s}(3, T 1)\right)\right\} \\
=\frac{2}{3} \max \left\{p^{s}(1,3), p^{s}(1,2), p^{s}(3,1), p^{s}(1,1), p^{s}(3,2)\right\} \\
=\frac{2}{3} \max \left\{2, \frac{3}{2}, 2,0, \frac{3}{2}\right\}=\frac{4}{3} .
\end{gathered}
$$

Since $L>R$, the conclusion follows.

The following example shows that there exist mappings that satisfy the contractive condition (5), but do not satisfy the contractive condition (28).

Example 12. Let $X=[0,2]$ be endowed with the partial metric

$$
p(x, y)= \begin{cases}|x-y| & \text { if } x, y \in[0,1], \\ \max \{x, y\} & \text { if }\{x, y\} \cap(1,2] \neq \varnothing .\end{cases}
$$

Clearly, $(X, p)$ is a 0 -complete partial metric space. Let $T, f$ : $X \rightarrow X$ be defined by

$$
T x= \begin{cases}x & \text { if } x \in[0,1] \\ \frac{1+x}{2} & \text { if } x \in(1,2]\end{cases}
$$

and $f x=x$ for each $x \in X$. As $T$ and $f$ have many common fixed points (each $x \in[0,1]$ is a common fixed point), then it is immediate to show that $T$ and $f$ do not satisfy the contractive condition (28).

If $(X, p)$ is ordered by

$$
x \preceq y \Longleftrightarrow(x=y) \text { or }(x, y \in(1,2], x \leq y) \text {, }
$$

then $T$ and $f$ satisfy the contractive condition (5) where $\psi$ : $[0,+\infty) \rightarrow[0,+\infty)$ is defined by

$$
\psi(t)= \begin{cases}\frac{t}{2} & \text { if } t \in[0,1], \\ \frac{1+t}{2} & \text { if } t \in(1,2] .\end{cases}
$$

Using Theorem 5, we deduce that $T$ and $f$ have a common fixed point.

\section{Completeness in Partial Metric Spaces and Fixed Points}

In this section, we characterize those partial metric spaces for which every Bianchini mapping has a fixed point in the style of Subrahmanyam characterization of metric completeness. This will be done by means of the notion of 0 -completeness which was introduced by Romaguera in [50].

Let $(X, p)$ be a partial metric space and $T: X \rightarrow X$ be a mapping. We recall that $T$ is a Bianchini [60] mapping if

$$
p(T x, T y) \leq k \max \{p(x, T x), p(y, T y)\}
$$

for all $x, y \in X$, where $0 \leq k<1$.

Theorem 13. Let $(X, p)$ be a partial metric space. If every mapping $T: X \rightarrow X$ satisfying the following conditions:

(i) $p(T x, T y) \leq \lambda \max \{p(x, T x), p(y, T y)\}$ for all $x, y \in$ $X$, for a fixed $\lambda>0$,

(ii) $T X$ is countable

has a fixed point, then $(X, p)$ is 0 -complete.

Proof. Suppose that there is a 0-Cauchy sequence $\left\{x_{n}\right\}$ of distinct points in $(X, p)$ which is not convergent in $\left(X, p^{s}\right)$. We put $A=\left\{x_{n}\right\}$ and we note that for every $x \notin A$, we have $p^{s}(x, A)>0$.

Now, $0<p^{s}(x, A) \leq 2 p(x, A)$ implies that $p(x, A)>0$. Since $\left\{x_{n}\right\}$ is a 0 -Cauchy sequence in $(X, p)$, there exists a least positive integer $N(x)$ such that

$$
\begin{aligned}
& p\left(x_{m}, x_{n}\right) \\
& \quad<\lambda p(x, A) \leq \lambda p\left(x, x_{l}\right), \quad l \in \mathbb{N}, \quad \forall m, n \geq N(x) .
\end{aligned}
$$

In particular

$$
\begin{aligned}
& p\left(x_{m}, x_{N(x)}\right) \\
& \quad<\lambda p(x, A \backslash\{x\}) \leq \lambda p\left(x, x_{N(x)}\right), \quad \forall m \geq N(x) .
\end{aligned}
$$


For fixed $n \in \mathbb{N}$, since $p^{s}\left(x_{n}, A \backslash\left\{x_{n}\right\}\right)>0$ and so $p\left(x_{n}, A \backslash\right.$ $\left.\left\{x_{n}\right\}\right)>0$, there is $n^{\prime}(n)>n$ such that

$$
p\left(x_{m}, x_{n^{\prime}}\right)<\lambda p\left(x_{n}, A \backslash\left\{x_{n}\right\}\right) \leq \lambda p\left(x_{n}, x_{n^{\prime}}\right), \quad \forall m \geq n^{\prime} .
$$

Now, let $T: X \rightarrow X$ be defined by

$$
T x= \begin{cases}x_{N(x)} & \text { if } x \notin A, \\ x_{n^{\prime}} & \text { if } x\left(=x_{n}\right) \in A .\end{cases}
$$

From the definition of $T$, we deduce that $T$ satisfies the condition (ii). On the other hand, $T$ satisfies also the condition (i). In fact, (i) is verified by assuming $T x=x_{n}$ and $T y=x_{m}$, and noting that

$$
p\left(x_{m}, x_{n}\right)< \begin{cases}\lambda p(y, A \backslash\{y\}) \leq \lambda p(y, T y) & \text { if } n \geq m, \\ \lambda p(x, A \backslash\{x\}) \leq \lambda p(x, T x) & \text { if } n<m .\end{cases}
$$

It is clear that $T$ has not fixed points since $x_{n^{\prime}} \neq x_{n}, n=1,2, \ldots$. Thus, the assumption that there is a 0 -Cauchy sequence $\left\{x_{n}\right\}$ which is not convergent in $\left(X, p^{s}\right)$ leads to a contradiction to Theorem 13 and thereby establishes the same.

If in Theorem 13 we choose $\lambda \in[0,1)$, by Corollary 9 , we obtain the following characterization of 0 -completeness for partial metric spaces.

Theorem 14. A partial metric space $(X, p)$ is 0-complete if and only if every mapping $T: X \rightarrow X$ satisfying the following conditions:

(i) $p(T x, T y) \leq \lambda \max \{p(x, T x), p(y, T y)\}$ for all $x, y \in$ $X$, for a fixed $\lambda \in[0,1)$,

(ii) $T X$ is countable

has a fixed point.

In Theorem 14, the class of mappings satisfying (i) and (ii) can be replaced by the class of mappings satisfying (ii) and the following condition:

(i) $p(T x, T y) \leq \lambda[p(x, T x)+p(y, T y)]$ for all $x, y \in$ $X$, for a fixed $\lambda \in[0,1 / 2)$.

\section{Acknowledgment}

The authors are grateful to the editor and referees for their valuable suggestions and critical remarks for improving this paper. P. Vetro is supported by Università degli Studi di Palermo (Local University Project R.S. ex 60\%).

\section{References}

[1] S. G. Matthews, "Partial metric topology," Annals of the New York Academy of Sciences, vol. 728, pp. 183-197, 1994, Papers on General Topology and Applications.

[2] S. J. O'Neill, "Partial metrics, valuations and domain theory," Annals of the New York Academy of Sciences, vol. 806, pp. 304-315, 1996, Papers on General Topology and Applications.
[3] M. A. Bukatin and J. S. Scott, "Towards computing distances between programs via Scott domains," in Logical Foundations of Computer Science, S. Adian, A. Nerode et al., Eds., vol. 1234 of Lecture Notes in Computer Science, pp. 33-43, Springer, Berlin, Germany, 1997.

[4] M. A. Bukatin and S. Y. Shorina, "Partial metrics and cocontinuous valuations," in Foundations of Software Science and Computation Structures, M. Nivat, Ed., vol. 1378 of Lecture Notes in Computer Science, pp. 125-139, Springer, Berlin, Germany, 1998.

[5] S. Romaguera and M. Schellekens, "Partial metric monoids and semivaluation spaces," Topology and Its Applications, vol. 153, no. 5-6, pp. 948-962, 2005.

[6] R. Heckmann, "Approximation of metric spaces by partial metric spaces," Applied Categorical Structures, vol. 7, no. 1-2, pp. 71-83, 1999.

[7] R. D. Kopperman, S. G. Matthews, and H. Pajoohesh, "What do partial metrics represent?" in Proceedings of the 19th Summer Conference on Topology and Its Applications, University of Cape'Town, 2004.

[8] H. P. A. Künzi, H. Pajoohesh, and M. P. Schellekens, "Partial quasi-metrics," Theoretical Computer Science, vol. 365, no. 3, pp. 237-246, 2006.

[9] S. Romaguera and M. Schellekens, "Duality and quasinormability for complexity spaces," Applied General Topology, vol. 3, no. 1, pp. 91-112, 2002.

[10] S. Romaguera and O. Valero, "A quantitative computational model for complete partial metric spaces via formal balls," Mathematical Structures in Computer Science, vol. 19, no. 3, pp. 541-563, 2009.

[11] M. P. Schellekens, "A characterization of partial metrizability: domains are quantifiable," Theoretical Computer Science, vol. 305, no. 1-3, pp. 409-432, 2003.

[12] M. P. Schellekens, "The correspondence between partial metrics and semivaluations," Theoretical Computer Science, vol. 315, no. 1, pp. 135-149, 2004.

[13] P. Waszkiewicz, "Partial metrisability of continuous posets," Mathematical Structures in Computer Science, vol. 16, no. 2, pp. 359-372, 2006.

[14] P. Waszkiewicz, "Quantitative continuous domains," Applied Categorical Structures, vol. 11, no. 1, pp. 41-67, 2003.

[15] V. Berinde and F. Vetro, "Common fixed points of mappings satisfying implicit contractive conditions," Fixed Point Theory and Applications, vol. 2012, article 105, 2012.

[16] L. J. Ćirić, “On contraction type mappings," Mathematica Balkanica, vol. 1, pp. 52-57, 1971.

[17] M. Abbas, T. Nazir, and S. Romaguera, "Fixed point results for generalized cyclic contraction mappings in partial metric spaces," Revista de la Real Academia de Ciencias Exactas, vol. 106, pp. 287-297, 2012.

[18] T. Abdeljawad, "Fixed points for generalized weakly contractive mappings in partial metric spaces," Mathematical and Computer Modelling, vol. 54, pp. 2923-2927, 2011.

[19] T. Abdeljawad, E. Karapinar, and K. Tas, "Existence and uniqueness of common fixed point on partial metric spaces," Applied Mathematics Letters, vol. 24, pp. 1894-1899, 2011.

[20] T. Abdeljawad, E. Karapinar, and K. Tas, "A generalized contraction principle with control functions on partial metric spaces," Computers \& Mathematics with Applications, vol. 63, pp. 716-719, 2012. 
[21] I. Altun and A. Erduran, "Fixed point theorems for monotone mappings on partial metric spaces," Fixed Point Theory and Applications, vol. 2011, Article ID 508730, 10 pages, 2011.

[22] I. Altun, F. Sola, and H. Simsek, "Generalized contractions on partial metric spaces," Topology and Its Applications, vol. 157, no. 18, pp. 2778-2785, 2010.

[23] H. Aydi, "Fixed point theorems for generalized weakly contractive condition in ordered partial metric spaces," Journal of Nonlinear Analysis and Optimization: Theory and Applications, vol. 2, pp. 33-48, 2011.

[24] H. Aydi, "Common fixed point results for mappings satisfying $(\psi, \phi)$-weak contractions in ordered partial metric spaces," International Journal of Mathematics and Statistics, vol. 12, pp. 53-64, 2012.

[25] H. Aydi, E. Karapinar, and W. Shatanawi, "Coupled fixed point results for $(\psi, \varphi)$-weakly contractive condition in ordered partial metric spaces," Computers \& Mathematics with Applications, vol. 62, no. 12, pp. 4449-4460, 2011.

[26] H. Aydi, M. Abbas, and C. Vetro, "Partial Hausdorff metric and Nadler's fixed point theorem on partial metric spaces," Topology and Its Applications, vol. 159, no. 14, pp. 3234-3242, 2012.

[27] H. Aydi, "A common fixed point result by altering distances involving a contractive condition of integral type in partial metric spaces," Demonstratio Mathematica. In press.

[28] K. P. Chi, E. Karapinar, and T. D. Thanh, "A generalized contraction principle in partial metric spaces," Mathematical and Computer Modelling, vol. 55, pp. 1673-1681, 2012.

[29] L. J. Ćirić, B. Samet, H. Aydi, and C. Vetro, "Common fixed points of generalized contractions on partial metric spaces and an application," Applied Mathematics and Computation, vol. 218, no. 6, pp. 2398-2406, 2011.

[30] C. Di Bari and P. Vetro, "Fixed points for weak $\varphi$-contractions on partial metric spaces," International Journal of Engineering, Contemporary Mathematics and Sciences, vol. 1, pp. 5-13, 2011.

[31] C. Di Bari, Z. Kadelburg, H. K. Nashine, and S. Radenović, "Common fixed points of g-quasicontractions and related mappings in 0-complete partial metric spaces," Fixed Point Theory and Applications, vol. 2012, article 113, 2012.

[32] D. Ilić, V. Pavlović, and V. Rakočević, "Some new extensions of Banach's contraction principle to partial metric space," Applied Mathematics Letters, vol. 24, no. 8, pp. 1326-1330, 2011.

[33] D. Ilić, V. Pavlović, and V. Rakočević, "Extensions of the Zamfirescu theorem to partial metric spaces," Mathematical and Computer Modelling, vol. 55, pp. 801-809, 2012.

[34] E. Karapinar, "Weak $\phi$-contraction on partial metric spaces," Journal of Computational Analysis and Applications, vol. 14, no. 2, pp. 206-210, 2012.

[35] E. Karapinar, "A note on common fixed point theorems in partial metric spaces," Miskolc Mathematical Notes, vol. 12, no. 2, pp. 185-191, 2011.

[36] E. Karapinar, "Generalizations of Caristi Kirk's theorem on partial metric spaces," Fixed Point Theory and Applications, vol. 2011, article 4, 2011.

[37] E. Karapınar and M. Erhan, "Fixed point theorems for operators on partial metric spaces," Applied Mathematics Letters, vol. 24, no. 11, pp. 1894-1899, 2011.

[38] E. Karapınar and U. Yüksel, "Some common fixed point theorems in partial metric spaces," Journal of Applied Mathematics, vol. 2011, Article ID 263621, 16 pages, 2011.

[39] H. K. Nashine, Z. Kadelburg, and S. Radenović, "Common fixed point theorems for weakly isotone increasing mappings in ordered partial metric spaces," Mathematical and Computer Modelling. In press.

[40] S. Oltra and O. Valero, "Banach's fixed point theorem for partial metric spaces," Rendiconti dell'Istituto di Matematica dell'Università di Trieste, vol. 36, no. 1-2, pp. 17-26, 2004.

[41] D. Paesano and P. Vetro, "Suzuki's type characterizations of completeness for partial metric spaces and fixed points for partially ordered metric spaces," Topology and Its Applications, vol. 159, no. 3, pp. 911-920, 2012.

[42] S. Romaguera, "Fixed point theorems for generalized contractions on partial metric spaces," Topology and Its Applications, vol. 159, no. 1, pp. 194-199, 2012.

[43] S. Romaguera, "Matkowski's type theorems for generalized contractions on (ordered) partial metric spaces," Applied General Topology, vol. 12, no. 2, pp. 213-220, 2011.

[44] B. Samet, "Coupled fixed point theorems for a generalized Meir-Keeler contraction in partially ordered metric spaces," Nonlinear Analysis, vol. 72, no. 12, pp. 4508-4517, 2010.

[45] B. Samet, M. Rajovic, R. Lazovic, and R. Stoiljkovic, "Common fixed point results for nonlinear contractions in ordered partial metric spaces," Fixed Point Theory and Applications, vol. 2011, article 71, 2011.

[46] W. Shatanawi, B. Samet, and M. Abbas, "Coupled fixed point theorems for mixed monotone mappings in ordered partial metric spaces," Mathematical and Computer Modelling, vol. 55, pp. 680-687, 2012.

[47] N. Shobkolaei, S. M. Vaezpour, and S. Sedghi, "A common fixed point theorem on ordered partial metric spaces," Journal of Basic and Applied Scientific Research, vol. 1, pp. 3433-3439, 2011.

[48] O. Valero, "On Banach fixed point theorems for partial metric spaces," Applied General Topology, vol. 6, no. 2, pp. 229-240, 2005.

[49] F. Vetro and S. Radenović, "Nonlinear $\psi$-quasi-contractions of Ćirić-type in partial metric spaces," Applied Mathematics and Computation, vol. 219, no. 4, pp. 1594-1600, 2012.

[50] S. Romaguera, "A Kirk type characterization of completeness for partial metric spaces," Fixed Point Theory and Applications, vol. 2010, Article ID 493298, 6 pages, 2010.

[51] W. A. Kirk, "Caristi's fixed point theorem and metric convexity," Colloquium Mathematicum, vol. 36, no. 1, pp. 81-86, 1976.

[52] J. Caristi and W. A. Kirk, "Geometric fixed point theory and inwardness conditions," in The Geometry of Metric and Linear Spaces, vol. 490 of Lecture Notes in Mathematics, pp. 74-83, Springer, Berlin, Germany, 1975.

[53] P. V. Subrahmanyam, "Completeness and fixed-points," Monatshefte für Mathematik, vol. 80, no. 4, pp. 325-330, 1975.

[54] S. Park, "Characterizations of metric completeness," Colloquium Mathematicum, vol. 49, no. 1, pp. 21-26, 1984.

[55] T. Suzuki, "A generalized Banach contraction principle that characterizes metric completeness," Proceedings of the American Mathematical Society, vol. 136, no. 5, pp. 1861-1869, 2008.

[56] M. Abbas and G. Jungck, "Common fixed point results for noncommuting mappings without continuity in cone metric spaces," Journal of Mathematical Analysis and Applications, vol. 341, no. 1, pp. 416-420, 2008.

[57] P. Vetro, "Common fixed points in cone metric spaces," Rendiconti del Circolo Matematico di Palermo, vol. 56, no. 3, pp. 464-468, 2007.

[58] S. Reich, "Some remarks concerning contraction mappings," Canadian Mathematical Bulletin, vol. 14, pp. 121-124, 1971. 
[59] L. J. Ćirić, "A generalization of Banach's contraction principle," Proceedings of the American Mathematical Society, vol. 45, pp. 267-273, 1974.

[60] R. M. T. Bianchini, "Su un problema di S. Reich riguardante la teoria dei punti fissi," Bollettino dell'Unione Matematica Italiana, vol. 5, pp. 103-108, 1972. 


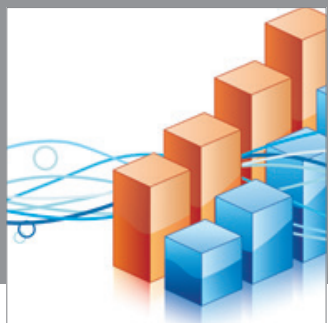

Advances in

Operations Research

mansans

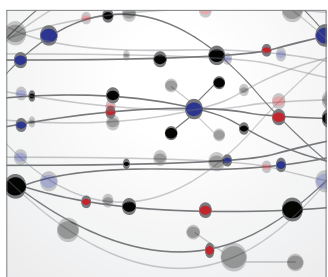

The Scientific World Journal
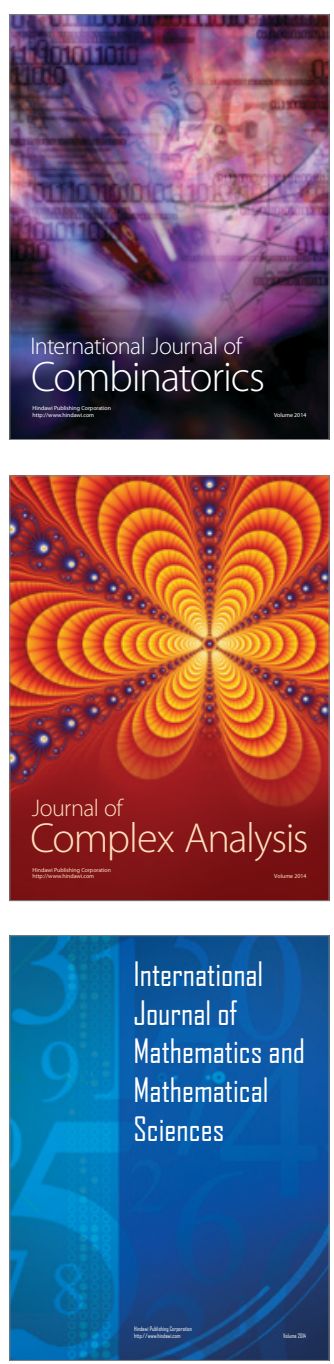
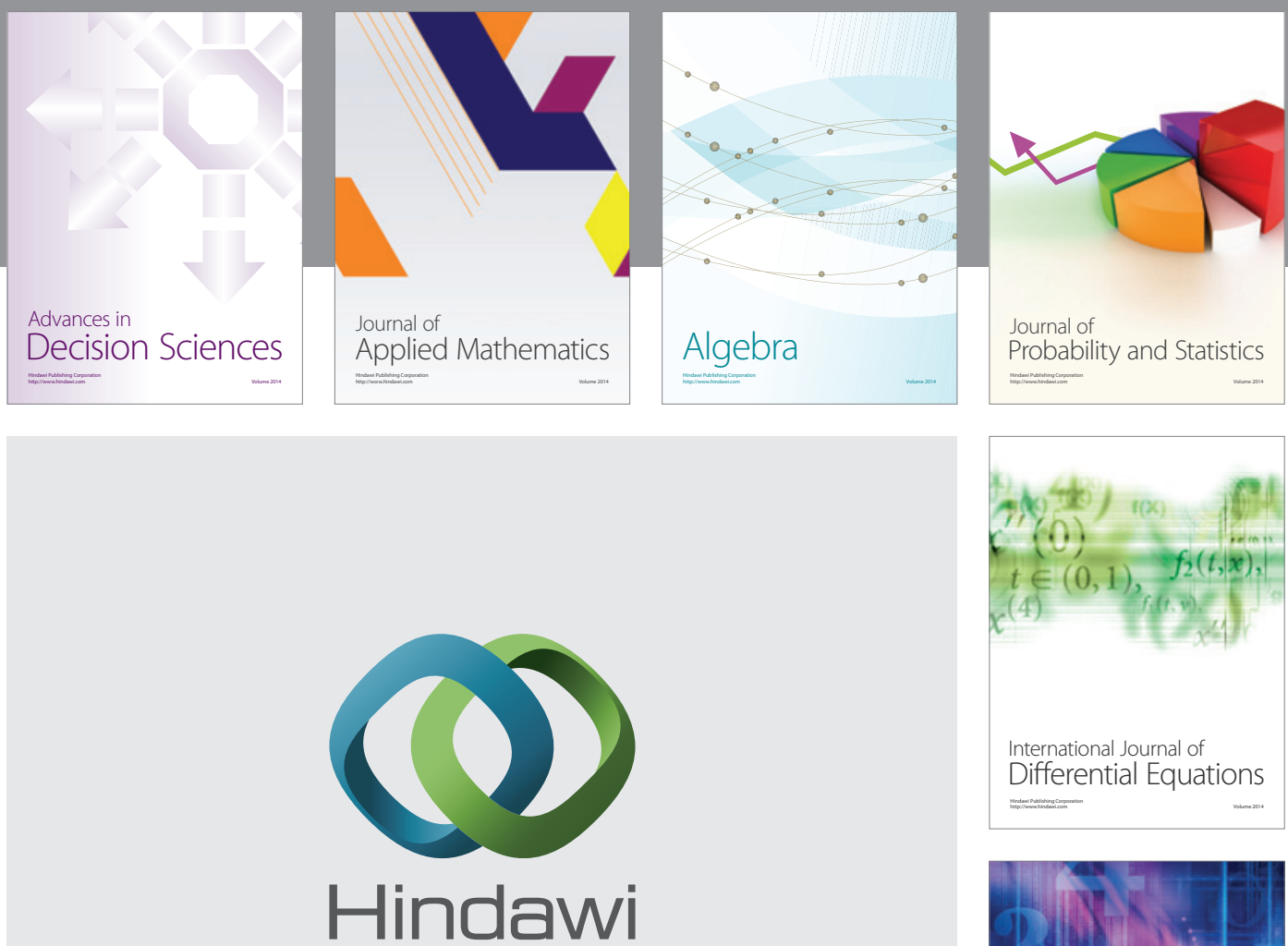

Submit your manuscripts at http://www.hindawi.com
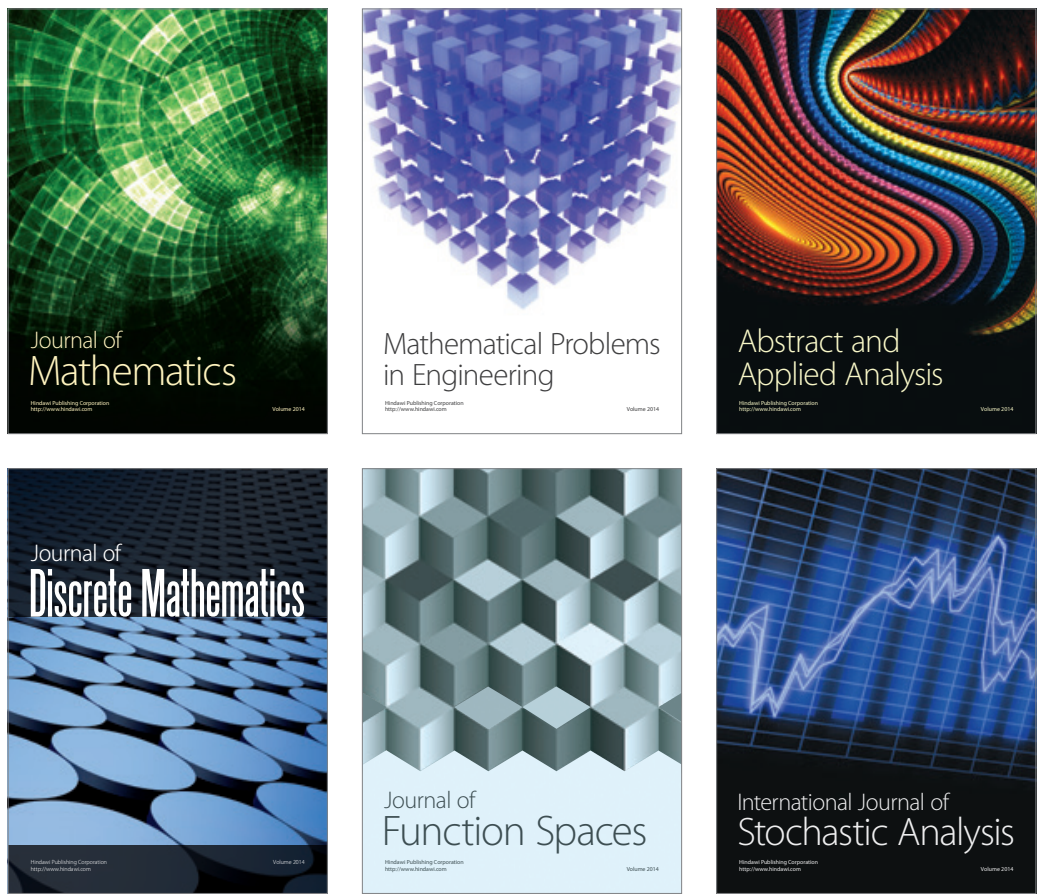

Journal of

Function Spaces

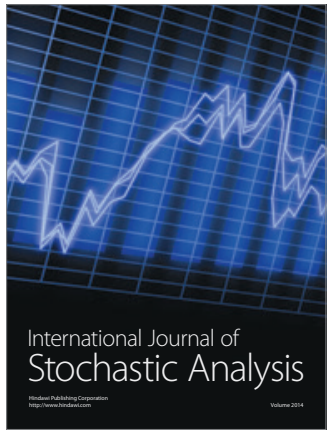

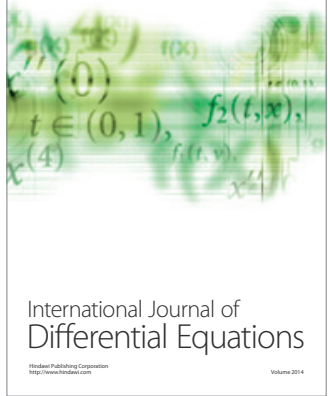
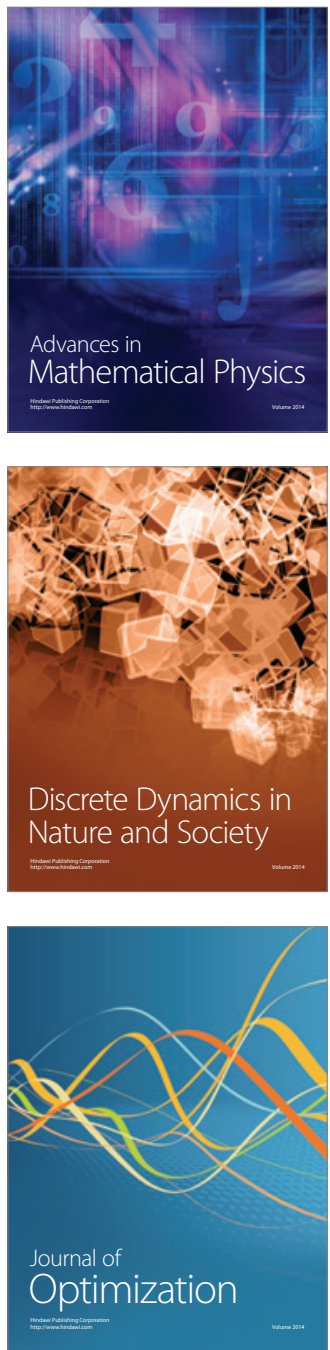